\title{
Una aproximación a la espiritualidad y carisma de Florencia Nightingale
}

\section{An approach to the spirituality and charism of Florence Nightingale Uma abordagem à espiritualidade e carisma de Florença Nightingale}

\author{
Francisco Ventosa Esquinaldo ${ }^{1}$
}

${ }^{1}$ Doctor en Enfermería. Director Centro Universitario de Enfermería San Juan de Dios. Bormujos, Sevilla. Correspondencia: Centro Universitario de Enfermería San Juan de Dios. Avda. San Juan de Dios s/n CP 41930, Bormujos (Sevilla). Correo electrónico de contacto: Francisco.ventosa@sjd.es

Cómo citar este artículo: Ventosa-Esquinaldo, F. (2021). Una aproximación a la espiritualidad y carisma de Florencia Nightingale. Cultura de los Cuidados. (Edición digital). 25 (No esp.). Recuperado de http://dx.doi.org/10.14198/cuid.2021.esp.09

Recibido:20/12/2020 Aceptado: 07/02/2021

\section{RESUMEN}

Interiorizar en los escritos de Florencia y de las autoras y autores que han escrito sobre ella, se descubren situaciones mágicas sobre su persona. La espiritualidad fue para ella una apertura a la realidad, a los demás y a la gran Alteridad que las religiones han designado con la palabra Dios. El carisma de Nightingale y su capacidad para influir en los demás comenzaron muy temprano en su vida. Ella se hizo presente donde la vida estaba destrozada, Crimea-Scutari, y allí es el lugar donde nace realmente "su carisma", comienza su ayuda a los que la necesitaban. Reflexionar hoy sobre la interioridad y la espiritualidad es necesario, es bueno, no está fuera de lugar. Florencia descubrió que los demás son importantes y no existen en función de los intereses personales, sino que solo desde la solidaridad es posible existir como seres humanos. Cuando Florencia Nightingale proyectó una escuela para la formación de enfermeras, el amor iba a ser la piedra angular sobre lo que el nuevo sistema sería construido. Con 80 años escribe a sus enfermeras y les habla de la grandeza de la profesión enfermera y le agradece su dedicación a su trabajo y por ser modelos para seguir. 
Palabras clave: Enfermería; cuidados; espiritualidad; carisma.

\section{ABSTRACT}

Internalize in the writings of Florence and the authors who have written about her, magical situations about her are discovered. Spirituality was for her an opening to reality, to others and to the great Alterity that religions have designated with the word God. Nightingale's charisma and ability to influence others began very early in his life. She was present where life was shattered, Crimea-Scutari, and there is the place where "her charisma" is really born, her help to those in need begins.

Reflecting today on interiority and spirituality is necessary, it is good, it is not out of place. Florencia discovered that others are important and do not exist based on personal interests, but that only from solidarity is it possible to exist as human beings. When Florence Nightingale planned a school for the training of nurses, love was to be the cornerstone on which the new system would be built. At the age of 80 , she writes to her nurses and talks to them about the greatness of the nursing profession and thanks them for their dedication to their work and for being role models.

Keywords: Nursing; care; spirituality; charisma.

\section{RESUMO}

Internalizando nos escritos de Florence e dos autores que escreveram sobre ela, situações mágicas sobre ela são descobertas. A espiritualidade foi para ela uma abertura à realidade, aos outros e à grande Alteridade que as religiões designaram com a palavra Deus. O carisma e a habilidade de Nightingale de influenciar outras pessoas começaram muito cedo em sua vida. Ela esteve presente onde a vida se despedaçou, Crimeia-Scutari, e é aí que nasce realmente "o seu carisma", começa a sua ajuda aos necessitados.

Refletir hoje sobre interioridade e espiritualidade é necessário, é bom, não está fora do lugar. Florencia descobriu que os outros são importantes e não existem por interesses pessoais, mas que só da solidariedade é possível existir como seres humanos. Quando Florence Nightingale planejou uma escola para o treinamento de enfermeiras, o amor seria a pedra angular sobre a qual o novo sistema seria construído. Aos 80 anos, ela escreve para suas enfermeiras e fala sobre a grandeza da profissão de enfermagem e as agradece por sua dedicação ao trabalho e por serem modelos.

Palavras-chave: Enfermagem; cuidados; espiritualidade; carisma.

\section{INTRODUCCIÓN}

La participación en este Congreso virtual ha supuesto en mí, interiorizar en una parte de sus escritos y de autores que han escrito sobre ella, con lo cual me siento muy satisfecho por los descubrimientos tan mágicos sobre la persona de Florencia Nightingale. Una aproximación a la espiritualidad y carisma de Florencia Nightingale. Es mi deseo intentar hacerles partícipes de esta realidad en su vida.

Los tiempos humanos no se determinan exclusivamente por las medidas del tiempo físico que incluye categorías que involucran el temple personal, el carácter de la época, el medio social o el momento cultural.

Un acontecimiento no se limita a acontecer, sino que acontece con una relevancia concreta y, en función de esa relevancia, vuelven a organizarse el pasado y el futuro.

Forman parte de la condición humana distendida en el tiempo la posibilidad y la necesidad de reinterpretar el pasado y 
replantearse el futuro en virtud de los acontecimientos que tienen lugar en el presente.

La época en la que se vive es la que prepara el futuro: los valores que son fundamento de nuestro testimonio encierran la semilla del futuro. Porque el compromiso $\mathrm{y}$ el testimonio no deben trasladarse constantemente a un futuro hipotético que nos impediría asumir nuestras responsabilidades presentes. Detenerse continuamente a proyectar -a pensar- el futuro sin crear y producir lo nuevo puede colocarnos fuera de la historia.

Nuestra actitud ante el futuro a de ser eminentemente positiva, creadora. Y la creatividad, como nos enseña la actual Filosofía de la Historia, solo se da cuando el hombre y la mujer en el presente asume activamente las posibilidades que le ofrece el pasado, y las pone en juego para crear un futuro más ajustado al ser humano.

\section{DESARROLLO DEL TEMA}

\section{Espiritualidad}

Espiritualidad no es religión, es la vida del espíritu. La dimensión espiritual es lo que alimenta la vida de los seres humanos. Lo espiritual existe en todos los seres humanos, se puede bloquear, pero con grandes pérdidas personales. La espiritualidad es, también creencias. La religión es un plus que se añade a la espiritualidad para construir caminos, tradiciones, mensajes y prácticas que conducen a la transformación de las personas y las llevan a la felicidad (Eseverri Chavarri, 2006).

Florencia comprendió y vivenció que la espiritualidad es una invitación a descentrarnos, a no encerrarnos en nosotros mismos, a salir de los egoísmos, a trascender nuestra individualidad e integrarnos en algo mayor, en algo que nos supera.

La espiritualidad fue para ella una apertura a la realidad, a los demás y a la gran Alteridad que las religiones han designado con la palabra "Dios". Es estar pendientes de lo real para darnos cuenta de los flujos que nos alimenta, interpelan o sostienen. Implica descubrir que no estamos solos y que no podemos vivir aislados, desentendiéndonos de cuanto no nos proporciona ningún beneficio directo (Otón Catalá, 2019).

Su camino espiritual le exigió vivir en verdad, ser sincera con ella misma, con Dios y con los demás. Lo falso es enemigo de lo espiritual (Otón Catalá, 2018).

En estos componentes se evidencia cómo la diversidad de aspectos que la componen hace de la espiritualidad un concepto amplio que no se puede limitar al aspecto religioso, $\mathrm{y}$ que, al ser claramente definidos, engrandecen la visión que se tiene del ser humano (Galvis López \& Pérez Giralvo, 2013).

A partir de este momento, Comenzó a construir su propio puente entre lo espiritual y el mundo practico. Como ella lo describió, "el reino de los cielos está en nuestro interior, de hecho, pero también debemos crear uno fuera, porque estamos destinados a actuar sobre nuestras circunstancias". Esta relación específica entre experiencia espiritual y práctica era poco común, incluso en la sociedad abiertamente religiosa de la Inglaterra victoriana. Nightingale resumió su doble enfoque en el misticismo y la acción, en una palabra: su deber.

El deber de Nightingale le dio la visión y la fuerza necesarias para su vida de servicio. Este sentido de su dirección nunca pareció abandonarla, incluso cuando enfrentó desafíos severos (Mongomery Dossey, Selanders, Beck, \& Attewell. 2005).

Florencia era seria, sensitiva, más bien callada y soñadora. En sus cartas de 
adolescente dice que Dios la llamó a su servicio, es el primer indicio de lo que será más tarde una vocación. Su juventud fue un largo y penoso conflicto interior en busca de su vocación y de la manera de satisfacerla. En el periodo que toda mujer decide casarse, también le llegó a Florencia, y nos habla de sus pretendientes y lo que compartirían, observamos que su amado ya estaba con ella y todo lo demás lo sentía como intrusos, y así vemos como en su vida fue fiel a este amor y como le fue fiel sirviéndole toda su vida.

Cuando estuvo tres meses con las Diaconisas fundadas por el Pastor Fliedner en Kaiserwerth, nos dice: ahora sé lo que es vivir y amar la vida... Dios en verdad ha hecho la vida rica en interés y en bendiciones y no deseo otra tierra, otro mundo sino este.

Al final de su ensayo titulado: Sugestiones para el pensamiento, dice:

- He revisado todas mis creencias religiosas desde el principio hasta el fin.

- He aprendido a conocer a Dios

- He rehecho mi credo social: ambos están escritos y listos para usar cuando mi hora haya llegado.

Su hora llegó; esa hora tan esperada y para la cual había vivido purificando su espíritu y adiestrando su inteligencia. La guerra de Crimea (Lewis, 1936).

El día 21 de octubre de 1854 partió con sus enfermeras con destino a Scutari, y llegaron el 4 de noviembre. Los médicos no daban crédito a la llegada de esta dama de buena familia en aquel infierno (Ayuso, 2007).

Florencia permanecería imperturbable ante la tenaz oposición y rudos ataques. La disciplina interior a la que se había sometido toda su vida la sostuvo. Ella lo expresa así:

¿Qué era yo frente al trabajo de mi Maestro? Cuando la gente ofende, antes que a mi ofende al Maestro. ¿Y quien soy yo para no soportar lo que a mi Maestro le place soportar? (Lewis, 1936).

Una espiritualidad desde la fragilidad humana que deposita el protagonismo de la propia vida en las manos de Dios no resulta ser mediocre. Todo lo contrario: transparenta la fuerza de Cristo al dejarlo actuar, a Su manera, mediante la propia vida (Mifsud, 2019).

Florencia contempló la importancia de la espiritualidad en el cuidado. Para ella, la espiritualidad es intrínseca a la naturaleza humana y es el recurso más profundo y potente de sanación del que dispone la persona (Galvis López \& Perez Giralvo, 2013)

Para Florencia, Dios había creado la enfermedad de las miasmas para que el hombre pudiera conocer sus causas a través de la observación del entorno. Creía, por consiguiente, que las enfermeras, al encargarse de la higiene, tenían una oportunidad para progresar espiritualmente, para descubrir la naturaleza de Dios mediante el aprendizaje de sus "leyes de la salud” (Attewell, 2010).

En su libro Notas sobre Enfermería. Que es y que no es, dice: A una cosa no le hace ser buena el que esté claro que una mujer sea capaz de hacerlo. Como tampoco le hace ser mala el que, siendo buena hecha por un hombre, haya sido hecha por una mujer.

Abandonemos estas pamplinas y vayamos decididas a hacer la obra de Dios con corazón limpio y sencillo (Nightingale, 1990).

El bienestar y la salud espiritual de los soldados a ella confiados, fue una preocupación constante y lo expresaba con sus desvelos: adecuándoles una sala para lectura, una escuela, escribiéndoles cartas de consuelo a sus madres cuyos hijos habían 
muerto, o para dales noticias de su mejoría. Los soldados agradecían su dedicación a ellos. Un soldado escribió: si nos dijeran que el techo se había abierto y que visiblemente había sido llevada al cielo, no nos hubiera sorprendido (Lewis, 1936).

Las cartas de Nightingale contienen conceptos profundos que describen una espiritualidad universal y la vida santa vivida en comunión con Dios y dedicada al servicio de Dios y a la sociedad. Ella articula su visión espiritual, que teje en su filosofía a través de ejemplos, parábolas, historias y ejemplos de atención de sus experiencias como enfermera en Harley Street, Scutari y Crimea (Mongomery Dossey, Selanders, Beck \& Attewell, 2005).

$$
\text { Nightingale no abordó }
$$
específicamente el lugar de la espiritualidad en la salud del individuo. Debido a que su propia espiritualidad estaba tan íntimamente ligada a su percepción de las personas y sus derechos inherentes a la atención en salud, es un elemento implícito más que manifiesto en su descripción de las personas (Mongomery Dossey, Selanders, Beck \& Attewell, 2005).

\section{Carisma}

Cuando se dice Carisma tiene un significado espiritual, es decir, una gracia, un poder, generalmente de tipo espiritual, un don concedido gratuitamente por Dios.

La psicología social en situaciones laicas la utiliza para indicar que alguien con esta cualidad puede influir en otras personas a nivel personal o de grupo (Ventosa Esquinaldo, 2012).

El carisma es una cualidad que posee una persona que destaca de las demás personas corrientes y por la que se le considera dotada de poderes y cualidades excepcionales. Así define el diccionario Carisma (Vazquez \& Vega Oncins, 1995).
Carisma es como un encanto benéfico de algo misterioso que nos acoge, nos moldea en el sublime servicio de cuidar, y nos va revelando la veracidad de nuestro propio ser y su exigencia de realizarnos siempre siendo de verdad lo que somos (Sánchez Martínez, 2004).

El carisma no es solo misión, aunque sin misión no hay carisma. El carisma que no se concreta socialmente, se queda en buenas ideas o palabras. Un carisma no visible, sin resonancia social, no es carisma, y todo lo que tiene una resonancia social se puede medir, y por tanto puede mejorar.

El hombre o mujer carismáticos hace primero la experiencia y, conforme ve que es posible llevar a cabo esa empresa, escribe. Primero se hace vida. No hay norma hay vida (Ventosa Esquinaldo, 2012). El carisma es finito -delimitado- en cuanto que necesita identificarse, es ilimitado en cuanto en cuanto que es imprevisto en sus manifestaciones. El carisma no es algo estático, sino en continuo desarrollo (Varona Alonso, 2010).

No es fácil hablar del carisma de Florencia. Pero conocemos su biografía y disponemos de sus escritos, y vemos su fuerza por mejorar la penosa situación de los enfermos, enriqueció la sensibilidad y el talante de los médicos y de aquellas mujeres cuidadoras de aquel momento. Ella aportó siempre lo mejor, dejando en sus escritos las pautas a seguir en las personas enfermas y las condiciones de una adecuada asistencia. Significar en ella su ímpetu, su vivencia de valores junto a los enfermos que ha trascendido y cambió esa forma de cuidar. La permanencia y transmisión de su herencia hasta hoy, le llamamos carisma (Sánchez Martínez, 2004).

Florencia Nightingale, creyente ahonda desde la fe en el ser del hombre y mujer y ve que, lejos de contradecir la 
ciencia, puede enriquecerla con una nueva visión. Establece con la persona que sufre una relación de ayuda insospechada, ilumina el acompañamiento en sus necesidades con un calor nuevo, y vive en comunión con él la profundidad cristiana de su empatía (Sánchez Martínez, 2004).

Enfermería desde su historia siempre han sido los valores cristianos los que han configurado su hacer $\mathrm{y}$, en el momento actual, si la enfermería quiere ser fiel a su historia, ha de incorporarlos; a su hacer técnico profesional ha de aunar los valores humanos que se esperan de ella. Entre ellos, como fundamental, el amor, expresado en acogida, comprensión, cercanía, compasión, respeto, que son los que aproximan a la persona, y los que el ser humano reclama cuando la enfermedad, el sufrimiento, y la soledad invaden su persona (Hernández Martín, 2011).

En sus escritos, que podríamos decir, en su vida, mucha firmeza de espíritu, no poca ironía y humor en sus juicios, pero, sobre todo, una profunda sensatez y clarividencia para ordenar lógicamente el confuso mundo de los cuidados de enfermería (Nightingale, F. 1990). Siempre estaba en su lugar, con una actitud sosegada y serena y dictaba las ordenes necesarias o hablaba de manera reconfortable. Prestando la atención necesaria, nada le resultaba demasiado pequeño o insignificante, $y$ toda su alma estaba plenamente entregada a su trabajo (Pollard, 2010).

Nada que afecte a los pobres le es indiferente e interviene en todo aquello que piensa pueda mejorar el bienestar de las personas, convencida de que su acción formaba parte de un plan elaborado por Dios para el progreso de la humanidad. Decide trabajar para ayudar a las prostitutas a encontrar otras salidas. Argumenta que no había que castigar a los pobres, sino enseñarles a que se valieran por si mismos, y que era, importante enseñarles una actividad, un oficio práctico que les permitiera ganarse la vida (Ayuso, 2007).

Revisando sus escritos, podemos sentir qué profundamente se identifica con aquellos a los que ayuda; haciendo suyas sus dificultades, suyas también, sus decepciones. Es precisamente esta certeza de ser plenamente comprendida lo que añade valor a su enseñanza (Pollard, 2010). En su código moral no había lugar para sentimentalismos; el trabajo, el amor y el conocimiento era a lo que recurría como ejemplo para aquellos que querían seguir sus pasos (Pollard, 2010).

Cuando conversaba con la religiosa Madre Coloma en sus visitas al convento romano de la Trinitá dei Monti en Roma, se interesaba especialmente por el proceso de formación de las novicias, particularmente cómo despertar y que medios para alimentar una vocación. Intuimos que años más tarde debió poner en práctica lo aprendido con su capacidad de encender en otros ese estado de ánimo en el cual una corriente fluye y lleva todo el ser a un fin superior (Lewis, 1936).

En 1853 le encargan que organice y dirija un Sanatorio para atender señoras. Inicia su trabajo el día 12 de agosto. En este difícil empeño aprendió a emplear todos los recursos desde la sugestión sutil, hasta la amenaza más o menos velada. Como dirigente sabía ser implacable y sabía prodigar ternura a las enfermas.

Esta situación fue motivada cuando el Comité del Sanatorio pretende imponer que solo sean admitidas pacientes anglicanas. Su actitud hace que esta norma sea cambiada, no será un impedimento la religión que tengan las pacientes para ser admitidas y atendidas (Lewis, 1936).

Una consecuencia importante de este impulso integrador se expresa en la superación de la dicotomía entre amor y 
Revista científica de la Asociación de Historia y Antropología de los Cuidados (Universidad de Alicante)

justicia. El amor exige la justicia, es su condición de posibilidad. La justicia a su vez alcanza su plenitud en el amor, que hace ver en cada uno de los seres humanos la presencia del amor creador y redentor de Dios. El amor supera con creces el plano de la justicia (Torralbe i Roselló, 2004).

El carisma de Nightingale y su capacidad para influir en los demás comenzaron muy temprano en su vida. Una de las personas que experimentó su carisma y genio fue Sidney Herbert en 1847, quien más tarde fue Secretario de Guerra en 1854 invitó a Nightingale a Crimea como Superintendente de enfermeras (Mongomery Dossey, Selanders, \& Attewell, 2005).

Ella se hizo presente donde la vida estaba destrozada, Crimea- Scutari, y allí es el lugar donde nace realmente "su carisma", comienza su ayuda a los que la necesitaban. $\mathrm{Su}$ personalidad y su carisma hizo que muchas normativas anacrónicas, que entorpecían organizar buenos cuidados, fueran obviadas. Se ha visto cuando dirige el Sanatorio para señoras, a su llegada a Scutari y durante toda su vida.

Valoraba la lógica por encima de cualquier sentimiento personal o de los demás. Era tan lógica y franca en sus opiniones que su veracidad a veces anulaba su tacto (Mongomery Dossey, Selanders, Beck \& Attewell, 2005).

Una de tantas acciones creativas en el desarrollo de su carisma era, adelantar gastos con su propio dinero y recuperarlo más tarde. Esto le llevó a implantar medidas administrativas demostrando sus conocimientos de experta administradora. Las enfermeras debían introducir la higiene y la disciplina en las salas. No estaban allí para la limpieza, sus funciones eran más significativas, y ellas debían crearlas. Florencia tanto en el viaje como una vez llegadas a Scutari las atendía en sus necesidades y les servía personalmente.
Ellas decían: nunca se tuvo tal cuidado de nuestras comodidades, nunca nos han tratado así, no sabíamos que miss Nightingale nos serviría (Lewis, 1936).

\section{CONCLUSIÓN}

Reflexionar hoy sobre la interioridad y la espiritualidad es necesario, es bueno, no está fuera de lugar. Florencia no vivió de espaldas a su interioridad y por tanto no estuvo sujeta a dependencias ni limitaciones. Ella descubrió y alimentó su dimensión interior transformando en sujeto su propia acción, responsable, libre del falso mundo de la imagen y la opinión exterior (Otón Catalá, 2018)

Descubrió que los demás son importantes y no existen en función de los intereses personales, sino que solo desde la solidaridad es posible existir como seres humanos. Este descubrimiento le implicó aprender que el amor no es una apetencia al servicio del propio egoísmo, sino un descentramiento (Otón Catalá, 2018).

Florencia pudo ver que la ciencia y el misticismo están irrefutablemente conectados y que la curación requiere atención al cuerpo, la mente, el espíritu y el medio ambiente. También reconoció la primacía del amor en la curación y que el servicio a la humanidad es el servicio a la fuente de la vida, ya sea que la fuente se llame Dios, Diosa, el Absoluto o el Bien Superior (Mongomery Dossey, B. Selanders, Beck, \& Attewell, 2005).

Cuando Florencia proyectó una escuela para la formación de enfermeras, el amor iba a ser la piedra angular sobre lo que el nuevo sistema sería construido. El amor implica sacrificio, pero no es visible, a veces incluso apenas se siente. La alegría de dar y el poder de implicación son el fruto del verdadero amor, siendo este de tan delicada 
textura, tan hermoso, que aporta una infinita alegría tanto al que da como al que lo recibe (Pollard, 2010).

A lo mejor piensa usted que estas cosas son pequeñeces, $\mathrm{o}$ al menos exageradas. Pero lo que usted "piensa”, o lo que "pienso" yo, importa poco. Veamos lo que Dios piensa de ellas. Dios siempre justifica sus caminos. Mientras que nosotros estamos pensando, El está enseñando. Sí, Dios siempre justifica sus caminos (Nightingale, 1990).

Escribe en 1860: Me pareció extraño, que se rezara para librarse de la peste, la peste y la hambruna cuando todas las alcantarillas comunes corrían hacia el Támesis, y las fiebres frecuentaban tierras sin drenaje, y se podían señalar los distritos que visitaría el cólera. Pensé que cuando llegara el cólera, podríamos mejorar estas causas, no que Dios eliminara el cólera (Mongomery Dossey, Selanders, Beck \& Attewell, 2005).

A la edad de 80 años escribe a sus enfermeras y les habla de la grandeza de la profesión enfermera y les agradece su dedicación a su trabajo y por ser modelos a seguir, y les recuerda al Padre: Siempre mantengan el honor de esta honorable profesión. ;Te agradezco, y puedo decir que nuestro Padre Celestial te agradece por lo que haces! Levanta alto el estandarte real. No sufrirá pérdida, el estandarte real de la enfermería. Debería ganar a través de cada uno de ustedes. Ha ganado inmensamente a través de ti (Mongomery Dossey, Selanders, Beck \& Attewell, 2005).

Florencia Nightingale es considerada, con todo rigor la fundadora de la institucionalización y regulación de los estudios de la Enfermería Moderna Laica (Hernandez Conesa \& Beneit Montesinos, 2012).

\section{BIBLIOGRAFÍA}

Attewell, A. (2010). Florence Nightingale (1820-1910). Temperamentum, 11.

Ayuso, E. (2007). Florence Nightingale. Salamanca: Imprenta Kadmos.

Diccionario Enciclopédico Olympia Ediciones. (1995). Madrid.

Eseverri Chávarri, C. (2006). Enfermería Facultativa. Madrid: Edt. Díaz de Santos.

Galvis López, A.M. y Pérez Giralvo, B. (2013). Revisión de la literatura sobre el concepto "espiritualidad" aplicado a la práctica de enfermería. Enfermería 21, 3(3). Hernández Conesa, J. y Beneit Montesinos, J.V. (2012). Un estudio de los paradigmas educativos enfermeros durante el siglo XIX. Murcia: Diego Marín Librero Editor.

Hernández Martín, F.J. (2011). De la norma a la responsabilidad. Hacia una ética de la responsabilidad de la enfermera. Madrid: Universidad Complutense de Madrid.

Lewis, J. T. (1936). Florencia Nightingale. Rosario- Argentina.

Mifsud, T. (2018-2019). Una espiritualidad desde la fragilidad. Dossier de Formación Permanente. Hnos. de S Juan de Dios. Madrid: Orden Hospitalaria de San Juan de Dios.

Mongomery Dossey, B., Selanders, L., Beck, D-M. y Attewell, A. (2005). Florence Nightingale Today. Maryland: American Nurses Association.

Nightingale, F. (1990). Notas sobre enfermería. Que es y que no es. Barcelona: Salvat Editores.

Otón Catalá, J. (2018-2019). Interioridad, Espiritualidad, Silencio. Dossier Formación Permanente. Hnos. de S Juan de Dios. Madrid: Orden Hospitalaria de San Juan de Dios.

Otón Catalá, J. (2018). Interioridad y espiritualidad. Edt. Santander: Sal Terre.

Pollard, E. (2010). Florence Nightingale. Bilbao.

Sánchez Martínez, J. (2004). Luces y 
contenidos teológicos en el movimiento de cuidados paliativos. Granada: ArchivoMuseo San Juan de Dios.

Torralba i Reselló, F. (2004) No olvidéis la Hospitalidad. Madrid: PPC Boadilla del Monte. Varona Alonso, M A. (2010). Gestión Carismática. Una apuesta por la Gestión Estratégica. Madrid: Fundación San Juan de Dios.

Ventosa Esquinaldo, F. (2012). Pensamiento de San Juan de Dios y la Orden Hospitalaria y su relación con la Enfermería: Conceptos y Valores. Granada: Archivo-Museo San Juan de Dios.

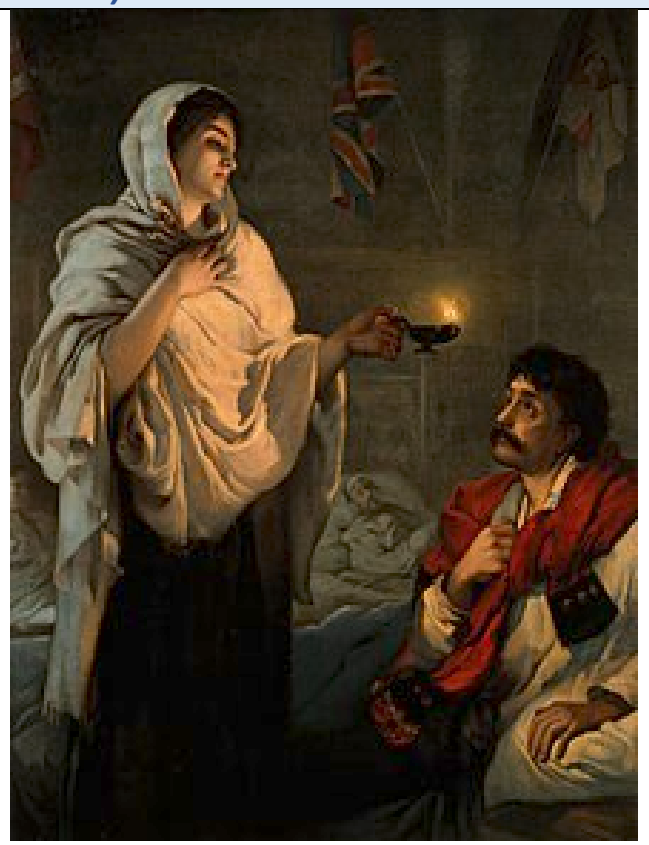

